\title{
Pilot study: can older inactive adults learn how to reach the required intensity of physical activity guideline?
}

This article was published in the following Dove Press journal:

Clinical Interventions in Aging

29 April 2013

Number of times this article has been viewed

\author{
Danielle R Bouchard ${ }^{1,2}$ \\ Marie-France Langlois ${ }^{3,4}$ \\ Katherine Boisvert-Vigneault ${ }^{3,4}$ \\ Paul Farand ${ }^{5}$ \\ Mathieu Paulin ${ }^{5}$ \\ Jean-Patrice Baillargeon ${ }^{3,4}$ \\ 'Faculty of Kinesiology and \\ Recreation Management, ${ }^{2} \mathrm{Health}$, \\ Leisure and Human Performance \\ Research Institute, University of \\ Manitoba, Winnipeg, Manitoba, \\ Canada; ${ }^{3}$ Departement of Medicine, \\ Division of Endocrinology, Université \\ de Sherbrooke, Sherbrooke, Quebec, \\ Canada; ${ }^{4}$ CRC Étienne LeBel, \\ Centre Hospitalier Universitaire de \\ Sherbrooke, Sherbrooke, Quebec, \\ Canada; ${ }^{5}$ Departement of Medicine, \\ Division of Cardiology, Université \\ de Sherbrooke, Quebec, Canada
}

Correspondence: Danielle R Bouchard Faculty of Kinesiology and Recreation Management, Health, Leisure and Human Performance Research Institute, 318 Max Bell Centre, R3T 2N2, University of Manitoba, Winnipeg, Manitoba, Canada

Tel +l 2044748627

$\mathrm{Fax}+|20426| 4802$

Email danielle_bouchard@umanitoba.ca

\begin{abstract}
Most individuals do not reach the recommended physical activity level of at least 150 minutes of aerobic exercise (AE) at moderate-to-vigorous intensity per week. For example, only $13 \%$ of older Canadian adults reach World Health Organization physical activity guideline (PAG). One of the reasons might be a difficulty identifying the required intensity. Twenty-five inactive older adults received one session about the AE-PAG and how to use a tool or strategy to help them identify AE intensity: heart-rate (HR) monitor ( $\%$ of maximal $\mathrm{HR} ; \mathrm{N}=9$ ); manual pulse ( $\%$ of maximal HR; $\mathrm{N}=8$ ); or pedometer (walking cadence; $\mathrm{N}=8$ ). Participants had 8 weeks to implement their specific tool with the aim of reaching the PAG by walking at home. At pre- and post-intervention, the capacity to identify AE intensity and AE time spent at moderateto-vigorous intensity were evaluated. Only the two groups using a tool increased total AE time (both $P<0.01$ ), but no group improved the time spent at moderate-to-vigorous intensity. No significant improvement was observed in the ability to correctly identify AE intensity in any of the groups, but a tendency was observed in the pedometer group $(P=0.07)$. Using walking cadence with a pedometer should be explored as a tool to reach the PAG as it is inexpensive, easy to use, and seemed the best tool to improve both AE time and perception of intensity.
\end{abstract}

Keywords: aging, pacing, aerobic exercise

\section{Introduction}

The World Health Organization (WHO) proposed a physical activity guideline (PAG) to optimize health, suggesting that people over 65 years of age accumulate 150 minutes of aerobic exercise (AE) at moderate-to-vigorous intensity per week. ${ }^{1}$ Despite the benefits associated with regular AE, only $13 \%{ }^{2}$ of older Canadian adults, aged 60 to 79 years old, currently reach the AE recommendations stated by the PAGs, and similar proportions are observed in other countries. ${ }^{1}$ It is not completely clear why older adults do not meet the minimum physical activity guidelines. However, it can be hypothesized that older adults overestimate what is considered "exercise." As a consequence, some social activities (eg, going to see friends) are seen as physical activities that count towards reaching the AE-PAG. This is supported by a systematic review that reports many studies have shown there is a discrepancy between self-reported and objective measures of intensity level. ${ }^{1,3}$ In addition, it is clear that older adults represent a major segment of the population that is considered inactive. ${ }^{2}$ Therefore, it is impossible to reach the guideline if one does not do a minimum of 150 minutes of aerobic exercise. Many older people are not familiar with the AE-PAG and this might also explain the low proportion who does not meet the guideline. ${ }^{4}$ 
While duration of a workout is easy to measure (eg, 30 minutes), the intensity of $\mathrm{AE}$ remains difficult to quantify. For example, it is easy to know that you went for a 30-minute walk, but it is hard to evaluate if the walk was done at moderate intensity. It is important to identify both intensity and duration when striving to achieve health benefits through $\mathrm{AE}^{5,6}$ as suggested by the WHO in the latest global recommendations for health. ${ }^{1}$ However, Schnohr et al ${ }^{7}$ indicate that walking intensity may have greater health significance than total walking duration in a follow-up of more than 10 years. For example, they reported that women who walked 30 to 60 minutes per day at high intensity decreased their mortality risk by $44 \%$ compared to only $12 \%$ for those who walked more than 2 hours per day at low intensity.

In the current literature, attempts to educate people in identifying $\mathrm{AE}$ intensity can be found in cardiac rehabilitation programs. ${ }^{8}$ However, the treatments have been shown to be unsuccessful in helping cardiac patients reach similar intensity once the program was completed at home. One of the reasons reported for not reaching the recommended intensity after the program was the absence of tools (eg, heart-rate [HR] monitor) to help them to identify the correct intensity as they had in the supervised program. ${ }^{9}$ In the past, studies attempted to determine whether people could identify moderate-to-vigorous intensity by comparing self-perceived exertion scores with their $\mathrm{HR}^{10-15}$ and reported low to moderate associations depending on the methodology used.

The first objective of this study was to determine whether inactive older adults were able to identify AE intensity. The second objective was to determine whether a combination of training regarding the AE-PAG and the use of different inexpensive strategies (ie, HR monitor, pedometer, manual pulse) would increase participants' ability to identify moderate-to-vigorous intensity and increase the number of participants reaching the AE-PAG after the study. It was hypothesized that older adults learning the appropriate $\mathrm{AE}$ intensity based on beats per minute or steps per minute would increase their ability to identify AE considered as moderate intensity. It was also hypothesized that such strategies would increase the total AE time spent at moderateto-vigorous intensity.

\section{Materials and methods Participants}

A sample of 25 older adults aged 65 years and older was recruited through newspapers or by word of mouth. Individuals were considered eligible to participate if they wanted to increase their $\mathrm{AE}$ level, but were currently doing less than 150 minutes of aerobic exercise at moderateto-vigorous intensity. Those who had a pacemaker, were currently using beta-blockers or any medication affecting HR at rest, or had participated in a program in which exercise intensity was discussed (eg, cardiac rehabilitation program) were excluded from the study. The ethics review board of Université de Sherbrooke, Sherbrooke, Quebec, Canada for research in humans approved the study protocol and written informed consent was obtained from each participant. After inclusion in the study, participants were randomly assigned to one of three groups: (1) manual pulse ( $\mathrm{N}=8)$; (2) pedometer (YAMAX Health and Sports, Inc., San Antonio, TX, USA $[\mathrm{N}=8]$ ); or (3) HR monitor (Polar Accurex Plus; Polar Electro, Woodbury, NY, USA [N = 9]).

\section{Training}

Each intervention group was provided with one separate halfday training session, comprising 3 hours of theory and 1 hour for practice with their assigned tool or strategy. The first part of the training session aimed to explain the AE-PAG. ${ }^{1}$ Resistance training and flexibility recommendations were briefly mentioned, but the emphasis was placed on the AE recommendations (ie, duration, intensity, and modalities of training) necessary to reach the minimum 150 minutes of $\mathrm{AE}$ at moderate-to-vigorous intensity weekly. General goals were given to participants. All participants were instructed to reach the minimal intensity every time they were doing physical activity for a minimum of 10 minutes at a time. In terms of duration, their objective was to increase it to a minimum of 75 minutes per week after 4 weeks and a minimum of 150 minutes after 8 weeks. Strategies to reach these goals were specified when each participant detailed a specific, measurable, attainable, realistic, and time-sensitive (SMART) goal for the next eight weeks. SMART goals are used to guide people to establish objectives. Each participant had to set SMART goals in the 4-hour session in order to increase the number of minutes of exercise for the next 8 weeks, and to reach the minimum intensity using their provided tool or strategy. These goals were revised in a follow-up phone conversation 4 weeks after the information/ training session. Each participant was also provided with an exercise log book to promote exercise adherence. They were also provided with a list of locations and walking groups they could join (eg, mall, parks) specific to their home surrounding. In addition, those who were interested could exchange their contact information to walk together. The second part of the training provided information on the 
specific equipment provided to help them correctly identify the intensity of their exercise at home. The participants in the HR monitor group and the manual pulse-rate group were given their personal HR reserve (HRR) range to help achieve moderate-to-vigorous intensity ( $\geq 40 \%$ of HRR) ${ }^{16}$ while walking. The following equation was used to calculate HRR and HR range prescription: ${ }^{17}$

$$
\begin{gathered}
\text { (Target } \% \times[\text { Maximum HR }- \text { Resting HR] } \\
+ \text { Resting HR) } .
\end{gathered}
$$

For each participant, maximum HR was determined by highest HR value observed during the initial treadmill test or estimated by an equation: ${ }^{18}$

$$
(206.9-[0.67 \times \text { age }]) .
$$

The pedometer group was instructed to target 100 steps/minute to reach at least moderate intensity. ${ }^{19}$ The participants in the manual pulse-rate group were taught how to manually measure their pulse rate and reach $\geq 40 \%$ of HRR. Every group was given 30 minutes to walk on a flat surface to test their tool and ask questions of the staff (one researcher and two students undergraduate in exercise sciences). All participants had already had the opportunity to use the HR monitor or pedometer for their 7-day evaluation before having the information session. Therefore, no one was completely new to the use of the equipment. All participants were also instructed to pay attention to the self-perceived exertion in order to identify the correct intensity when not wearing the device at the end of the intervention. Each participant was contacted by phone after 4 weeks to increase compliance and answer questions in regard to the provided tool or strategy.

\section{Knowledge of the PAG}

At pre- and post-training, participants answered three questions on the PAG. The first question was: According to the PAG, for how long should a person above age $65 \mathrm{do}$ AE every week to achieve optimal health? The answer was reported in number of minutes. If participants reported a range, the lower number was recorded. The second question was: What is the minimum $\mathrm{AE}$ intensity to achieve optimal health for a person above age 65? The possible answers were "low," "moderate," "vigorous," or "don't know." The third question was: What is the recommended frequency of resistance training for a person above age 65 ? The participant was expected to answer a number of days per week.

\section{Measures of exercise level and intensity during a typical week}

All participants were asked to record all physical activities done in seven consecutive days with the use of a pedometer and a HR monitor pre- and post training. Note that no information on the PAG was given before baseline measurement. During that week, they were also instructed to $\log$ all physical activities lasting 10 minutes or more in duration. No instructions regarding the AE type, duration, or intensity were given at baseline. The pedometer was worn on the waist, while the participant was awake, and the total number of steps was recorded each day. The HR monitor was worn (chest strap and wristwatch) for every activity lasting more than 10 consecutive minutes. Afterwards, the recorded HR monitor files were downloaded with the provided Polar software. Data were used to calculate the total time and intensity for each reported activity.

\section{Self-reported exercise level}

The long version of the International Physical Activity Questionnaire (IPAQ) was used to assess activities across a variety of different domains, including leisure time, domestic work, and active transportation. ${ }^{20}$ Each domain assesses activities performed at moderate-to-vigorous intensity for at least 10 consecutive minutes for 7 days. An average metabolic equivalent (MET) score was calculated for total AE performed weekly. Individual MET scores for walking and moderate and vigorous activities were calculated within each domain and combined to provide a total score of MET minutes/week. The goal of using this tool was to verify the differences between self-reported exercise levels and objective measures of physical activity level.

\section{Perception of AE intensity during the treadmill test}

To our knowledge, there is no standardized measure to evaluate an individual's ability to correctly identify AE intensity. In this study, perception of exercise intensity was evaluated during a medically supervised treadmill test, using a modified version of the Cornell protocol, ${ }^{21}$ after a short familiarization session. The test started with a warm-up (speed $1.7 \mathrm{mph}$; slope fixed at $10 \%$ ) for 2 minutes. After, walking speed was increased by $0.4 \mathrm{mph}$ and the slope increased by $1 \%$ every 2 minutes. The test continued until the participant either reached predicted maximal HR (Equation 2) ${ }^{18}$ or exhaustion. After each minute, the participants were asked about their perception of $\mathrm{AE}$ intensity on the Borg scale (CR-10), ${ }^{22}$ and HR was noted. 
To measure a participant's ability to correctly identify moderate intensity, the number of minutes that heart rate reserve (HRR) was between $45 \%$ and 59\% and the Borg scale between 4 and 6 was divided by the total number of minutes that HRR was between $45 \%$ and 59\%. ${ }^{10,16}$ HRR was calculated as: ${ }^{16}$

$$
\text { Maximal HR - Resting HR × Intensity (\%), }
$$

while resting HR was measured with a blood pressure monitor (Omron HEM-711, Omron Healthcare Inc., Vernon Hills, IL, USA) after spending 5 minutes at rest. The proportion of well-identified moderate intensity represents the number of minutes that HRR between $45 \%$ and $59 \%$ and Borg scale between 4 and 6 corresponded (reporting HRR and Borg scale in moderate intensity at the same time) and was divided by the total number of times that HRR was between 4 and 6 .

\section{Other clinical measures}

Other clinical measures were collected to characterize the sample and are described in the following paragraphs.

Body mass was measured to the nearest $0.1 \mathrm{~kg}$ on a calibrated scale (SECA, Hamburg, Germany), and height was obtained with a standard stadiometer (Takei, Tokyo, Japan). Body mass index (BMI) was calculated by dividing body mass by height squared. Waist circumference (WC) was measured twice at the end of normal expiration using a measuring tape placed at the top of the iliac crest as suggested by the US National Institutes of Health. ${ }^{23}$ The mean of WC measurements was used for analyses. Percentage of fat mass was measured by foot-to-foot bioimpedance scale (Tanita scale C-300, Tanita Corporation of Americas, Inc, Arlington Heights, Illinois, USA), according to standard procedures. ${ }^{24}$ Blood pressure and resting HR were measured by a Critakon Dinamap Pro 100 Vital Signs Monitor (Johnson and Johnson, New Brunswick, NJ, USA).

Physical capacity was measured by two strength tests and a walking speed test. Maximum voluntary isometric strength of the knee extensor was measured using a belt resistance chair $^{25}$ assisted by a hand-held dynamometer (Microfet2 ${ }^{\text {TM}}$; Hoggan Health Industries, Draper, UT, USA). Three maximal contractions were recorded on each side in an alternating approach. Handgrip strength was measured with a JAMAR dynamometer (Preston, Jackson, MI, USA). Subjects were tested while sitting on a chair with their arm adducted, elbow flexed at a 90-degree angle with the hand in a neutral position. At the end of a normal respiration, subjects had to squeeze their hand at their maximal strength. For all three measurements, contractions were maintained for 4 seconds, with a rest of 30 seconds between each measure. The 6-minute walk test was used to assess physical capacity according to standard procedures. ${ }^{26}$ The result of this test is known to be associated with subsequent morbidity or mortality. ${ }^{27}$

Chronic conditions were reported at baseline using a modified version of the Older American Resources and Services questionnaire. ${ }^{28}$ The following chronic conditions were listed: arthritis, edema, asthma, emphysema, blood pressure, cardiac or circulation problems, diabetes, stomach ulcer, digestive chronic conditions, liver disease, urinary problem, osteoporosis, anemia, thrombosis, stroke, Parkinson's disease, muscular dystrophy, thyroid problem, and others. The participants were asked to answer "yes" (1) or "no" (0) if they currently had any of the chronic conditions listed above. The total number of chronic conditions was recorded as the sum of the number of positive answers.

\section{Statistical analyses}

Data management and statistical analyses were performed using SPSS version 17.0 (IBM Corporation, Armonk, NY, USA). Normality of variables was tested by Shapiro-Wilk test. Results are reported as mean \pm standard deviation for normally distributed variables. Median (25th-75th percentiles) was reported for variables not normally distributed, and N (\%) for categorical variables. Differences between pre- and post-intervention values within each group were evaluated using Wilcoxon signed-rank test or paired $t$-tests, as deemed appropriate. Differences among groups were evaluated by either Kruskal-Wallis or analysis of variance (ANOVA) tests, as deemed appropriate. To identify differences between the manual pulse group and the two other groups (using either the pedometer or the HR monitor), $t$-test or Mann-Whitney tests were used on pre- and post-changes. The alpha level was corrected by the number of statistical tests to minimize type I error. In subanalyses, men and women as well as two different age groups (65-70 and $\geq 70$ years old) were compared using Wilcoxon signed-rank test or paired $t$-tests on the study main outcomes.

\section{Results}

Participants' characteristics are presented in Table 1. At baseline, BMI and self-reported AE were different between groups (Table 1).

\section{Perception of AE intensity on the treadmill standardized test}

At baseline, the percentage of correctly identified exercise intensity was $25 \%, 23 \%$, and $10 \%$ in the manual pulse, pedometer, and HR monitor groups, respectively. 
Table I Participants' characteristics at baseline

\begin{tabular}{ll}
\hline Baseline characteristics & $\begin{array}{l}\text { Total } \\
(\mathbf{N}=\mathbf{2 5})\end{array}$ \\
\hline Age (years) & $71.9 \pm 4.5$ \\
Male sex & $12(48.0)$ \\
College or university level completed & $13(52.0)$ \\
Sum of chronic conditions & $3.3 \pm 1.6$ \\
Health risk factors & \\
Weight $(\mathrm{kg})$ & $71.7(65.2-97.6)$ \\
Body mass index $\left(\mathrm{kg} / \mathrm{m}^{2}\right)^{*}$ & $27.2(23.9-32.8)$ \\
Waist circumference $(\mathrm{cm})$ & $92.1(83.2-106.5)$ \\
Fat mass $(\%)$ & $32.4 \pm 9.1$ \\
Systolic blood pressure $(\mathrm{mmHg})$ & $129.9 \pm 22.9$ \\
Diastolic blood pressure $(\mathrm{mmHg})$ & $81.4 \pm 16.7$ \\
Physical capacity & \\
Leg strength $(\mathrm{kg})$ & $21.0 \pm 6.9$ \\
Handgrip strength $(\mathrm{kg})$ & $21.9(17.4-32.6)$ \\
6-minute walk $(\mathrm{m})$ & $51 \mathrm{I} \pm 82.0$ \\
\hline Nots: Data
\end{tabular}

Notes: Data are presented as unadjusted mean \pm standard deviation, $N(\%)$, or median (25th-75th percentile). $* P \leq 0.05$ for difference among groups at baseline.

The percentage of correctly identified exercise intensity increased in all groups, but the improvements did not reach statistical significance (Figure 1). Nonetheless, a greater improvement was observed in the pedometer group $(+15 \%)$ compared to the HR monitor group $(+10 \%)$ or the manual pulse group $(+7 \%)$.

\section{PAG knowledge}

At baseline, the minimum $\mathrm{AE}$ mean time per week that was reported by participants as the minimum required was more than the minimum recommended by the AE-PAG in all three groups (Table 2). After the intervention, the perception of the needed AE time per week, for optimal health benefits, was still higher than 150 minutes per week on average, with the exception of the pedometer group, who answered that, on average, duration of $138 \pm 73$ minutes per week should be the target. In terms of AE intensity, more than $88 \%$ of participants declared, when given multiple choices, that $\mathrm{AE}$ at moderate intensity should be the minimum target to reach optimal health benefits when exercising, both at baseline and post-intervention.

\section{Self-reported AE level and objective measures of $A E$}

At baseline, the HR monitor group reported doing more total $\mathrm{AE}$ and more $\mathrm{AE}$ at moderate intensity $(P<0.05)$ compared to other groups. However, only the pedometer group increased total $(P=0.003)$ and moderate-intensity reported $\mathrm{AE}$ level $(P=0.003)$ after the intervention. When exercise was objectively measured by wearing a blinded pedometer for 7 days, participants performed an average of

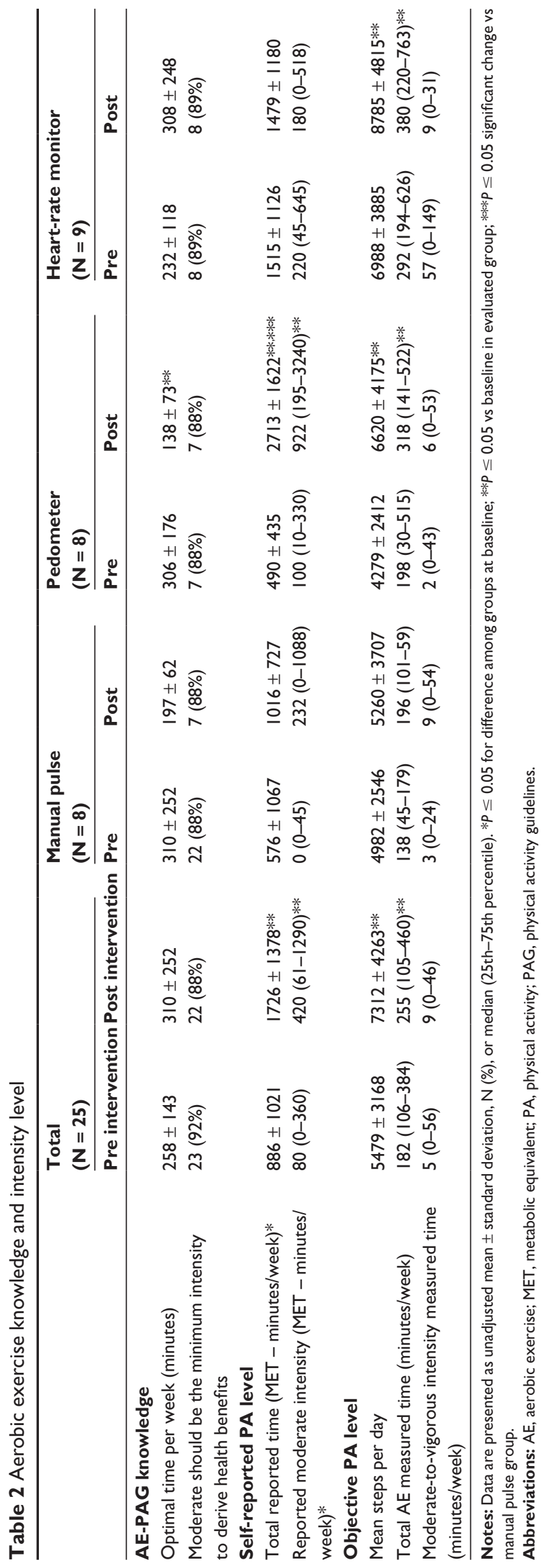




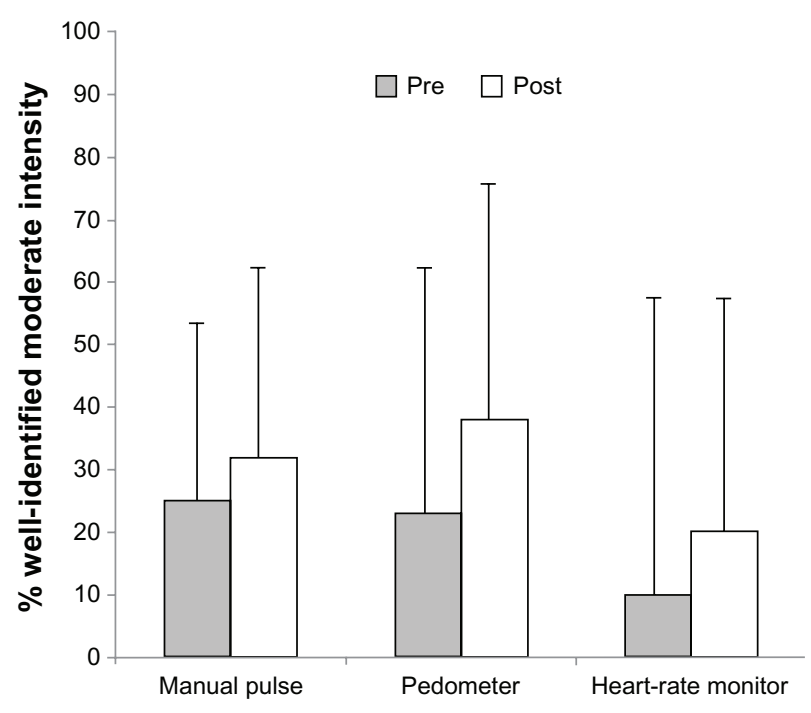

Figure I Exercise intensity correctly identified as moderate.

Notes: \% of well-identified moderate intensity represents the number of minutes that heart rate reserve (HRR) was between $45 \%$ and $59 \%$ and the Borg scale between 4 and 6 divided by the total number of minutes that HRR was between $45 \%$ and $59 \%$. Data are presented as median (25th-75th percentile).

$5479 \pm 3168$ steps per day at baseline. Mean steps per day increased in the two groups using instruments (pedometer: $35.0 \%$; $P<0.001$; HR monitor: $35.5 \%$; $P=0.003)$, but did not significantly increase in the group using manual pulse to monitor AE intensity $(5.3 \% ; P=0.26)$. In terms of total duration of AE recorded on the HR monitor, a mean total of 182 minutes was recorded at baseline without any difference between groups. The duration of $\mathrm{AE}$ recorded by the HR monitor increased to 255 minutes after the intervention in the whole sample $(28.7 \% ; P=0.05)$. Both groups using a tool (pedometer: $38.2 \%$; $P=0.04$; HR monitor: $23.2 \% ; P=0.03$ ) increased at the post-intervention compared to baseline, but no such improvement was observed in the manual pulse group. Finally, no change was noticed in the AE time spent at moderate-to-vigorous intensity in any group.

Finally, potential differences on the identification of moderate intensity and the number of minutes spent at moderate intensity between pre- and post-intervention were tested between men and women as well as between the two age groups ( $65-70$ and $\geq 70$ years old). No significant difference was observed.

\section{Discussion}

This study shows that the majority of our sample of older inactive adults did not reach the AE-PAG, and most were unable to correctly identify exercise intensity. Simple and inexpensive training accompanied by the use of either a pedometer or a HR monitor seems to be effective in increasing time devoted to $\mathrm{AE}$ in inactive older adults.
However, the same strategies, as tested in this study, seem insufficient to increase AE time spent at moderateto-vigorous intensity, or to improve the perception of $\mathrm{AE}$ intensity. Based on our findings, older adults monitoring steps per minute with a pedometer were more likely to reach a significant improvement in the ability to correctly identify moderate intensity.

The first aim of this study was to determine whether inactive older adults were able to identify AE intensity. Our results suggest that, in our sample, the answer was no, as the participants only correctly identified moderate intensity for a mean of $18 \%$ of the time based on the treadmill test. The second aim was to determine whether a combination of training regarding the AE-PAG and the use of different tools or strategies (ie, HR monitor, pedometer, manual pulse) would increase participants' ability to identify moderate-tovigorous intensity and increase the number of participants reaching the AE-PAG after the study. Although participants using a tool to monitor AE increased their ability to correctly identify moderate intensity during the treadmill tests, no significant improvement was observed, and this knowledge was not transferred in performing more AE-PAG compared to baseline.

Only the pedometer group increased their self-reported level of moderate-intensity $\mathrm{AE}$ and improved their knowledge regarding minimal weekly AE duration needed to obtain health benefits. In fact, this group answered that an average of $306 \pm 176$ minutes per week was necessary (or recommended) before the program's intervention, but reported an average of $138 \pm 73$ minutes per week after the intervention, which is closer to the AE-PAG. This might be explained by the fact that they assimilated the information that they had received during the half-day training about the AE-PAG. On the other hand, the two other groups overestimated the number of $\mathrm{AE}$ time to reach health benefits before as well as after the intervention. Finally, the pedometer group correctly identified moderate-intensity AE during the post-intervention evaluation more frequently than the other groups. Therefore, this study suggests that using a pedometer might be a better option when trying to help older inactive adults increase their level of $\mathrm{AE}$ at moderate intensity and the ability to correctly identify AE intensity. Our results suggest that walking cadence (steps per minute) might be easier to perceive than cardiovascular rhythm to reach the AE intensity stated by the PAG. Thus, with an attempt to increase the proportion of older adults who reach the AE-PAG, we propose that further research explores the benefits of interventions based on walking cadence using a 
pedometer to promote $\mathrm{AE}$ at moderate-to-vigorous intensity in older inactive adults.

At baseline evaluation, $92 \%$ of participants responded that moderate intensity while exercising was necessary to achieve health benefits. Most inactive older adults in our sample were thus aware that moderate-intensity exercise is needed to reach optimal health benefits through AE. More studies are needed to evaluate if our results can be applicable to the majority of older adults. This high percentage of our sample who knew that moderate intensity was the targeted intensity by the PAG is surprising, since a 2002 study suggested that only one-fifth of adults knew about the guidelines. ${ }^{29}$ However, our sample was highly educated, multiple-choice answers were provided, and the new AE-PAG was launched only a few days prior to recruitment, resulting in media coverage.

Our study shows that no significant increase in time devoted to AE at moderate intensity was observed after the intervention. First, it is possible that participants did not totally understand the home task, in part because too much information was given at the half-day training session, including the use of technology for the HR monitor group. Second, since inactive participants were selected, their main goal for participation in the study may have been to increase $\mathrm{AE}$ duration and not $\mathrm{AE}$ intensity. For those reasons, we believe that the proposed intervention could be improved by splitting the intervention into two separate phases: the first phase could aim to increase total AE duration (eg, progressively reach 150 minutes per week or more), and the second phase could focus on AE intensity.

Although Tudor-Locke et $\mathrm{al}^{30}$ used a different strategy to evaluate the association between steps per day and $\mathrm{AE}$ minutes spent at moderate or moderate-to-vigorous intensity, they reported that 7000 to 10,000 steps per day are generally sufficient to reach the AE-PAG in older adults. Therefore, it was expected that the mean steps per day observed in this study (7312 \pm 4263$)$ at post-intervention would be closely associated to 150 minutes AE at moderate intensity. However, only a median time of 9 minutes $(3.5 \%)$ of the total monitored $\mathrm{AE}$ was performed at moderate intensity during the evaluation week after the intervention. This small proportion is in line with the $13 \%$ reported on a recent national-scale study that objectively measured physical activity levels with an accelerometer. $^{2}$ This small proportion of weekly AE time spent at moderate intensity is in contrast with what was measured (by HR monitor) in total duration of AE during the 7-day evaluation. Consequently, for some older inactive adults included in our study, believed that many low-intensity activities such as shopping, house chores, or gardening were considered as AE time that counted towards the 150 minutes of $\mathrm{AE}$ recommended by the PAG. More importantly, those results imply that, when public promotions are focusing on the importance to reach at least 150 minutes of $\mathrm{AE}$ at moderate intensity, inactive older individuals may not feel targeted. Therefore, it is important to pursue strategies to inform older adults on the type of activity or exercise that is considered moderate intensity and on the importance of increasing their HR level.

Of note, our study results show that half-day training on the AE-PAG and the use of a pedometer or HR monitor is sufficient to increase total AE time. This result is in line with a review showing that it is possible to increase $\mathrm{AE}$ time in older adults with simple interventions. ${ }^{31}$ Although promising, the use of such strategies to improve the capacity to correctly identify AE intensity and increase AE at moderate intensity needs to be improved. For example, it is possible that adding a few exercise group sessions could help participants understand the task and the usage of tools.

Despite the promising results underlined in this pilot study, some limitations need to be addressed. Some differences were observed among groups at baseline such as BMI and activity level in the HR monitor group. This could be due to the small sample size, but it could have affected the possible improvement in AE level and intensity. In this pilot study, we did not control the exact time participants performed exercise and whether the participants actually used the respective equipment each time they exercised. If this was the case, we might have underestimated the number of AE minutes spent at moderate intensity. Participants learned how to identify moderate-tovigorous intensity in a natural environment, but were tested on a treadmill. Nonetheless, the present study addresses an important public health concern, and is strengthened by the exploration of different strategies using tools to improve $\mathrm{AE}$ intensity perception. Overall, this study consists of a step in the right direction to help older adults reach the PAG, and the next steps should focus on the pedometer as a tool to reach $\mathrm{AE}$ at moderate intensity based on individual cadence associated to HRR. Future studies aiming to increase the proportion of older adults reaching the AE-PAG should first aim to increase total $\mathrm{AE}$ duration and then focus on $\mathrm{AE}$ intensity, as one of the reasons for not having observed an increase in the time spent at moderate intensity could be the fact that simply doing something more was the participant's objective.

\section{Conclusion}

In summary, this study shows that older inactive adults self-report weekly AE above the minimum requested by the 
PAG, but only a small amount of this AE is performed at moderate intensity. Interestingly, half-day didactic training sessions on the AE-PAG and how to reach moderate intensity seems to be a promising intervention when associated with a pedometer using steps per minute (cadence) to estimate intensity. However, this strategy needs to be improved to increase the amount of moderate-intensity AE or the ability to identify moderate intensity.

\section{Disclosure}

The authors report no conflict of interest in this work.

\section{References}

1. Global recommendation on physical activity for health [webpage on the Internet]. Geneva: World Health Organization; 2010. Available from: http://www.who.int/dietphysicalactivity/factsheet_recommendations/ en/. Accessed February 13, 2013.

2. Colley RC, Garriguet D, Janssen I, Craig CL, Clarke J, Tremblay MS. Physical activity of Canadian adults: accelerometer results from the 2007 to 2009 Canadian Health Measures Survey. Health Rep. 2011; 22(1):15-23.

3. Prince SA, Adamo KB, Hamel ME, Hardt J, Connor Gorber S, Tremblay M. A comparison of direct versus self-report measures for assessing physical activity in adults: a systematic review. Int $J$ Behav Nutr Phys Act. 2008;5:56.

4. Plotnikoff RC, Lippke S, Johnson ST, Hugo K, Rodgers W, Spence JC. Awareness of Canada's Physical Activity Guide to Healthy Active Living in a large community sample. Am J Health Promot. 2011;25(5):294-297.

5. Manini TM, Everhart JE, Patel KV, et al. Daily activity energy expenditure and mortality among older adults. JAMA. 2006;296(2):171-179.

6. Williams PT. Vigorous exercise, fitness and incident hypertension, high cholesterol, and diabetes. Med Sci Sports Exerc. 2008;40(6): 998-1006.

7. Schnohr P, Scharling H, Jensen JS. Intensity versus duration of walking, impact on mortality: the Copenhagen City Heart Study. Eur J Cardiovasc Prev Rehabil. 2007;14(1):72-78.

8. Lear SA, Ignaszewski A. Cardiac rehabilitation: a comprehensive review. Curr Control Trials Cardiovasc Med. 2001;2(5):221-232.

9. Scotto CJ, Waechter D, Rosneck J. Patients' perception of monitoring during cardiac rehabilitation: taking the message home. J Nurs Care Qual. 2009;24(3):263-268.

10. Chen MJ, Fan X, Moe ST. Criterion-related validity of the Borg ratings of perceived exertion scale in healthy individuals: a meta-analysis. J Sports Sci. 2002;20(11):873-899.

11. Eston R, Connolly D. The use of ratings of perceived exertion for exercise prescription in patients receiving beta-blocker therapy. Sports Med. 1996;21(3):176-190.

12. Eston RG, Williams JG. Reliability of ratings of perceived effort regulation of exercise intensity. Br J Sports Med. 1988;22(4):153-155.

13. Hetzler RK, Seip RL, Boutcher SH, Pierce E, Snead D, Weltman A. Effect of exercise modality on ratings of perceived exertion at various lactate concentrations. Med Sci Sports Exerc. 1991;23(1):88-92.

Clinical Interventions in Aging

\section{Publish your work in this journal}

Clinical Interventions in Aging is an international, peer-reviewed journal focusing on evidence-based reports on the value or lack thereof of treatments intended to prevent or delay the onset of maladaptive correlates of aging in human beings. This journal is indexed on PubMed Central, MedLine, the American Chemical Society's 'Chemical Abstracts
14. Shephard RJ, Kavanagh T, Mertens DJ, Yacoub M. The place of perceived exertion ratings in exercise prescription for cardiac transplant patients before and after training. Br J Sports Med. 1996; 30(2):116-121.

15. Watt B, Grove R. Perceived exertion. Antecedents and applications. Sports Med. 1993;15(4):225-241.

16. Warburton DE, Nicol CW, Bredin SS. Prescribing exercise as preventive therapy. CMAJ. 2006;174(7):961-974.

17. Swain DP, Leutholtz BC. Heart rate reserve is equivalent to \%VO2 reserve, not to \%VO2 max. Med Sci Sports Exerc. 1997;29(3):410-414.

18. Gellish RL, Goslin BR, Olson RE, McDonald A, Russi GD, Moudgil VK. Longitudinal modeling of the relationship between age and maximal heart rate. Med Sci Sports Exerc. 2007;39(5):822-829.

19. Marshall SJ, Levy SS, Tudor-Locke CE, et al. Translating physical activity recommendations into a pedometer-based step goal: 3000 steps in 30 minutes. Am J Prev Med. 2009;36(5):410-415.

20. Craig CL, Marshall AL, Sjostrom M, et al. International physical activity questionnaire: 12-country reliability and validity. Med Sci Sports Exerc. 2003;35(8):1381-1395.

21. Hollenberg M, Ngo LH, Turner D, Tager IB. Treadmill exercise testing in an epidemiologic study of elderly subjects. J Gerontol A Biol Sci Med Sci. 1998;53(4):B259-B267.

22. Borg G. Borg's Perceived Exertion and Pain Scales. Human Kinetics. 1998. ISBN-10: 0880116234; ISBN-13: 978-0880116237.

23. [No authors listed]. Clinical guidelines on the identification, evaluation, and treatment of overweight and obesity in adults: executive summary. Expert Panel on the Identification, Evaluation, and Treatment of Overweight in Adults. Am J Clin Nutr. 1998;68(4):899-917.

24. Jebb SA, Cole TJ, Doman D, Murgatroyd PR, Prentice AM. Evaluation of the novel Tanita body-fat analyser to measure body composition by comparison with a four-compartment model. Br J Nutr. 2000; 83(2):115-122.

25. Kramer JF, Vaz MD, Vandervoort AA. Reliability of isometric hip abductor torques during examiner- and belt-resisted tests. $J$ Gerontol. 1991;46(2):M47-M51.

26. ATS Committee on Proficiency Standards for Clinical Pulmonary Function Laboratories. ATS statement: guidelines for the six-minute walk test. Am J Respir Crit Care Med. 2002;166(1):111-117.

27. Bautmans I, Lambert M, Mets T. The six-minute walk test in community dwelling elderly: influence of health status. BMC Geriatr. 2004;4:6.

28. Fillenbaum GG, Smyer MA. The development, validity, and reliability of the OARS multidimensional functional assessment questionnaire. J Gerontol. 1981;36(4):428-434.

29. Spence JC, Plotnikoff RC, Mummery WK. The awareness and use of Canada's Physical Activity Guide to Healthy Active Living. Can J Public Health. 2002;93(5):394-396.

30. Tudor-Locke C, Craig CL, Aoyagi Y, et al. How many steps/day are enough? For older adults and special populations. Int J Behav Nutr Phys Act. 2011;8(1):80.

31. Nelson ME, Rejeski WJ, Blair SN, et al; American College of Sports Medicine; American Heart Association. Physical activity and public health in older adults: recommendation from the American College of Sports Medicine and the American Heart Association. Circulation. 2007;116(9):1094-1105.

\section{Dovepress}

Service' (CAS), Scopus and the Elsevier Bibliographic databases. The manuscript management system is completely online and includes a very quick and fair peer-review system, which is all easy to use. Visit $\mathrm{http}: / / \mathrm{www}$.dovepress.com/testimonials.php to read real quotes from published authors. 